\title{
Tumor suppression by miR-31 in esophageal carcinoma is p21- dependent
}

\author{
Zhifeng Ning ${ }^{1,2,5}$, Hua Zhu8, Feifei Li' ${ }^{2,5}$, Qing Liu'2,5, Gefei Liu6 ${ }^{6}$ Tao Tan ${ }^{8}$, Bo Zhang ${ }^{8}$, \\ Shaobin Chen ${ }^{4}$, Guanwu $\mathrm{Li}^{7}$, Dongyang Huang ${ }^{6}$, Stephen J. Meltzer ${ }^{9}$ and Hao \\ Zhang $2,3,5$ \\ ${ }^{1}$ Laboratory for Translational Oncology basic medicine college, Hubei University of Science and Technology, Xianning, Hubei \\ province, China \\ ${ }^{2}$ Department of Biotherapy and Gastrointestinal Medical Oncology, Affiliated Cancer Hospital of Shantou University Medical \\ College, Shantou, Guangdong, China \\ ${ }^{3}$ Tumor Tissue Bank, Affiliated Cancer Hospital of Shantou University Medical College, Shantou, Guangdong, China \\ ${ }^{4}$ Department of Thoracic Surgery, Affiliated Cancer Hospital of Shantou University Medical College, Shantou, Guangdong, \\ China \\ ${ }^{5}$ Cancer Research Center, Shantou University Medical College, Shantou, Guangdong, China \\ ${ }^{6}$ Department of Cell Biology, Shantou University Medical College, Shantou, Guangdong, China \\ ${ }^{7}$ Department of Biochemistry, Shantou University Medical College, Shantou, Guangdong, China \\ 8 Department of Surgery, Davis Heart and Lung Research Institute, the Ohio State University Wexner Medical Center, \\ Columbus, $\mathrm{OH}$, USA \\ ${ }^{9}$ Division of Gastroenterology, Department of Medicine, The Johns Hopkins University School of Medicine and Sidney Kimmel \\ Comprehensive Cancer Center, Baltimore, MD, USA
}

Correspondence to: Hao Zhang, email: haozhang@stu.edu.cn

Keywords: microRNA, miR-31, p21, esophageal squamous cell cancer, personalized medicine

Received: March 27, $2014 \quad$ Accepted: October 08, $2014 \quad$ Published: October 09, 2014

This is an open-access article distributed under the terms of the Creative Commons Attribution License, which permits unrestricted use, distribution, and reproduction in any medium, provided the original author and source are credited.

\section{ABSTRACT}

microRNA regulation network is important for the cancer genetic heterogeneity. Relative to the increasing numbers of microRNA's targets identified, upstream regulatory mechanisms that control functional microRNAs are less well-documented. Here, we investigated the function of $\operatorname{miR}-31$, a pleiotropically-acting microRNA, in esophageal squamous cell cancer (ESCC). We demonstrated that miR-31 only exerted tumor-suppressive effects in TE-7 ESCC cells, but not in TE-1 ESCC cells, although both of these cell lines harbor inactive p53. Interestingly, TE-1 cells highly expressed p21, while p21 levels were virtually undetectable in TE-7 cells, suggesting a p21-dependent mechanism of miR-31-mediated tumor suppression. Accordingly, knockdown of p21 in TE-1 cells reversed the tumor suppressive actions of miR-31. In patient ESCC specimens, real-time RT-PCR analysis revealed that expression of E2F2 and STK40, two known miR-31 target oncogenes, was negatively correlated with the expression of miR-31 in a p21-dependent manner, supporting the conclusion that miR-31 only downregulates its target oncogenes when p21 levels are low. Collectively, these data suggest a novel mechanism through which the tumor-suppressive effect of miR-31 is p21-dependent. In addition, we speculate that delivery of miR-31 could provide therapeutic benefit in the personalized management of a subgroup of ESCC patients with p21-deficient tumors.

\section{INTRODUCTION}

Esophageal carcinoma ranks seventh in cancer incidence and sixth in cancer-related death worldwide, respectively, with esophageal squamous cell carcinoma (ESCC) accounting for $90 \%$ of all histological types of 
esophageal carcinomas diagnosed at advanced stages [13]. Even with improved surgical techniques, the median survival of ESCC patients after R0 resection (complete removal of the entire tumor, followed by microscopic examination of margins showing no tumor cells) is less than 2 years $[4,5]$. Difficulty in treatment of ESCC is partly attributed to genetic heterogeneity of the disease, which is in part due to complex regulatory networks and is the challenge for personalized therapy [6-9]. Thus, the identification of regulatory pathways for stratification of this deadly disease is critical to developing personalized and precision therapeutics and enhancing survival.

microRNA network is of importance for genetic heterogeneity of diseases. microRNAs (miRs) are endogenous single-stranded non-coding RNAs ranging from 19 to 25 nucleotides in length which play important roles in epigenetic and post-transcriptional regulatory networks [10]. Considerable evidence has identified the involvement in cancer of one of these RNAs, miR-31, but its role is complicated because it can act as either a tumor suppressor or an oncogene: while it inhibits cell proliferation of serious ovarian carcinomas [11] and cell metastasis in breast cancer [12], and impairs migration of endometrial cancer cells [13] and growth of prostate cancer in vivo [14], miR-31 also promotes tumorigenesis in colorectal cancer [15] and cell migration and invasion in Kaposi's sarcoma [16]. In ESCC, miR-31 has also been reported to be both a promoter and an inhibitor of carcinogenesis [17-20]. While microarray screening showed miR-31 to be upregulated in ESCC vs. normal epithelia $[17,18]$, patients with high miR-31 expression levels had an improved prognosis [20]. Further work suggested that miR-31-mediated downregulation of DNA repair genes contributes to an improved prognosis of ESCC patients after radiotherapy [19]. These findings suggested that the complex action of miR-31 might reflect genetic heterogeneity among ESCC patients.

The multifaceted role of miR-31 suggests that its action depends on cellular and molecular context. Relative to its known downstream targets, there is less knowledge regarding how miR-31 is regulated by upstream mechanisms or interactions with other molecules. Interestingly, effects of miR-31 have been linked to the status of p53, the most frequently mutated gene in all cancers. miR-31 plays an inhibitory role only in tumor cells harboring mutant p53, suggesting miR-31 as a therapeutic target in patients with p53-deficient tumors [11]. Of note, p53 mutation is an early signature event in ESCC; moreover, changes in p53 status could account for context-dependent effects of many molecules [21], including microRNAs such as miR-31 [11]. However, it is unknown whether there is an association between p53 status and miR-31 in ESCC. Clarity regarding cellular mechanisms accounting for miR-31's function in cancer will be beneficial in designing tailored diagnostic and therapeutic strategies for ESCC and other malignancies.
In this study, we attempted to study the molecular mechanism underlying miR-31-mediated inhibition of p53-deficient ESCC. Surprisingly, we found that while the ESCC cell lines TE-7 and TE-1 harbored deficient p53, miR-31 only exhibited tumor-suppressive activity in the p21-low-expressing cell line TE-7, and not in p21-high-expressing TE-1 cells. However, after p21 was silenced by shRNA, the suppressive function of miR31 was rescued in TE-1 cells. Moreover, we analyzed the correlation between miR-31 and its known target oncogenes, E2F2 and STK40, in 27 human ESCC tissues. As in our observations in cancer cell lines, the inhibitory effect of miR-31 on its targets was also p21-dependent. Our findings suggested a novel mechanism via which the tumor-suppressive function of miR-31 depends on p21 status, suggesting the p21-miR-31 pathway as a potential therapeutic target in a subgroup of ESCC patients.

\section{RESULTS}

\section{Differential effects of miR-31 in ESCC cell lines harboring inactive p53}

A previous study demonstrated that miR-31 functions as a tumor suppressor only in p53-deficient cells [11]. We tested this finding in several ESCC cell lines that harbored inactive p53, including TE-1 [22] and TE-7 [23]. After overexpression of miR-31 in TE-7 cells, cell viability assessed by MTT assay decreased dramaticantly $(p<0.05)$ (Fig. 1A), colony formation ability tested using plate colony formation assay decreased by $43 \%$ (Fig. 1B), and cell invasion evaluated by transwell assay decreased by over $46 \%$ (Fig. 1C).

To explore molecular mechanisms underlying miR-31-mediated tumor- suppression, we examined the expression of E2F2 and STK40, two known downstream target oncogenes for miR-31 [14, 24]. Transfection and forced overexpression of miR-31 reduced E2F2 and STK40 by real-time RT-PCR (Fig. 1D), suggesting that miR-31 may suppress ESCC by downregulating target oncogenes, including E2F2 and STK40.

To our surprise, transfection of miR-31 had no effect on TE-1 cells in terms of cell growth, colony formation, or invasion (Fig. 2A-C), nor did miR-31 downregulate E2F2 or STK40 (Fig. 2D). These results suggested that even though TE-1 cells harbor inactive p53, they express other factor(s) that inhibit miR-31 function.

\section{p21 inhibits the effects of miR-31}

Because miR-31-mediated tumor suppression was previously shown to depend on p53 deficiency, but our miR-31-resistant TE-1 cell line was also p53-deficient, we tested TE-1 and TE-7 cells for expression of the p53 target 


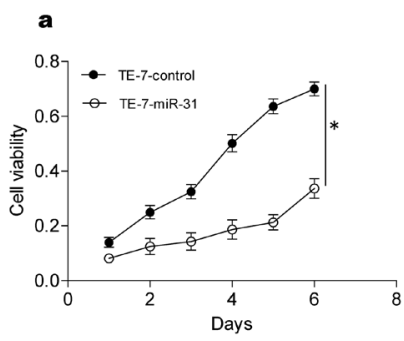

b
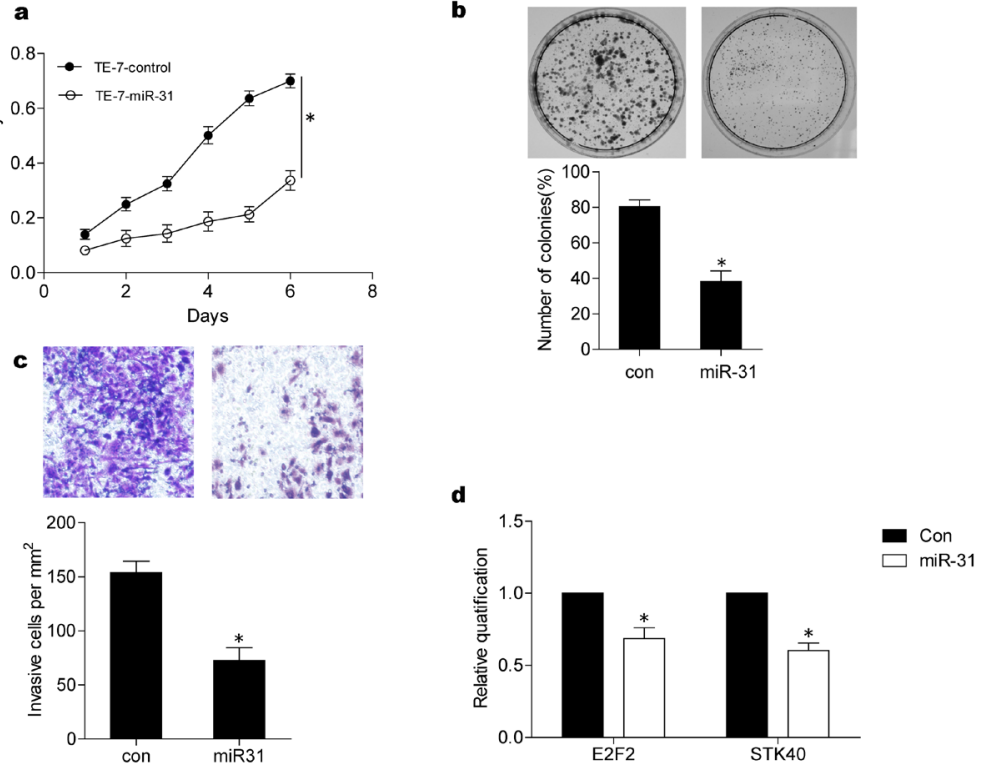

Figure 1: Tumor suppressive effects of miR-31 in TE-7 ESCC cells. (A) Cell viability was determined by MTT assay. Transfection of miR-31 inhibited cell viability of TE-7 cells. (B) miR-31 treatment inhibited colony formation capacity of TE-7 cells by about $43 \%$ as shown in the lower panel, a representative colony formation assay was shown (upper panel). (C) Invasive ability of TE-7 cells was inhibited over $90 \%$ by miR-31 treatment. Representative pictures of invaded cells (upper panels); cell number per $1 \mathrm{~mm}^{2}$ was counted under $100 \mathrm{X}$ magnification and summarized (lower panel). (D) The effects of miR-31 on E2F2 and STK40 were shown by real time RT-PCR. Transfection of miR-31 can inhibit mRNA levels of E2F2 and STK40. The data were the average of three independent experiments and presented as means \pm SEM. * indicating $\mathrm{P}<0.05$ con, control.
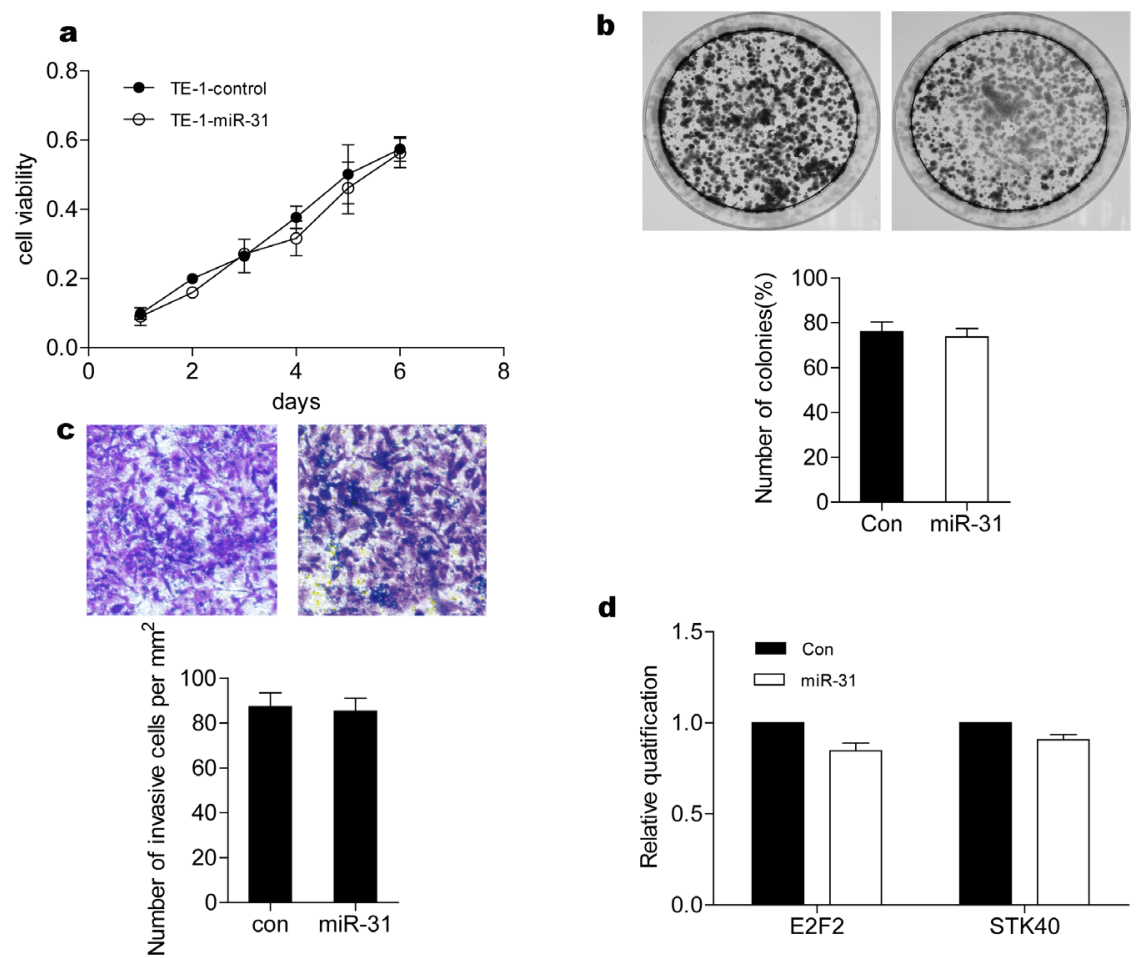

Figure 2: Lack of miR-31-mediated suppression in TE-1 ESCC cells. (A) Cell viability was determined by MTT assay. Transfection of miR-31 did not inhibit cell growth. (B) miR-31 treatment did not inhibit colony formation capacity of TE-1 cells (lower panel). The representative figures of stained colonies (upper panel). (C) TE-1 cells invasion was not inhibited by miR-31 treatment. Representative pictures of invaded cells (upper panels), cell number per $1 \mathrm{~mm}^{2}$ was counted under $100 \mathrm{X}$ magnification and summarized (lower panel). (D) Effects of miR-31 on E2F2 and STK40 were shown by real time RT-PCR. Transfection of miR-31 did not reduce E2F2 and STK40 mRNA levels. The data were the average of three independent experiments and presented as means \pm SEM with $*$ indicating $\mathrm{P}<0.05$. con, control. 
protein p21. p21 was highly expressed in TE-1 cells, but undetectable in TE-7 cells (Fig. 3), suggesting that p21 could be responsible for inhibition of miR-31-mediated tumor suppression. To test this hypothesis, we transfected p21-specific shRNA into TE-1 cells. As shown in Fig. $4 \mathrm{~A}, \mathrm{p} 21$ shRNA reduced endogenous expression of $\mathrm{p} 21$ by $85 \%$. Thus, if $\mathrm{p} 21$ inhibited tumor suppressor function of miR-31, one would expect that p21 shRNA treatment would sensitize TE-1 to miR-31. Indeed, miR-31 inhibited viability, colony formation, and invasion of TE-1 cells following shRNA-mediated knockdown of p21 (Fig. 4BD). Moreover, the suppressive effect of miR-31 on cell survival depended on silencing of p21 in TE-1 cells (Fig. 4B). Real-time RT-PCR also showed that miR-31 was able to downregulate the expression of E2F2 and STK40 after p21 shRNA treatment (Fig. 4E), further demonstrating that the tumor-suppressive effects of miR-31 were dependent on reduced p21 expression.

\section{Expression of p21, miR-31, and miR-31-target genes in ESCC patients}

In order to validate our in vitro findings in patients with ESCC, we collected biopsies from 27 ESCC patients and assessed the correlation of p21, miR-31, and miR-31target gene expression by real-time RT-PCR. Although expression of miR-31 and p21 fluctuated among the 27 ESCC tissues, there was no statistically significant correlation between expression of miR-31 and p21 in human ESCC biopsies (data not shown). However, miR31 expression was negatively correlated with E2F2 and STK40 in biopsies with low p21 expression (Fig. 5A). When p21 expression was high, miR-31 expression did not correlate with E2F2 and STK40 expression (Fig. 5B). These results suggested miR-31 regulates the expression of its target genes in p21-deficent ESCC human tissues. Taken together, the observations in human tumor biopsies confirmed our hypothesis that the tumor-suppressive effect of miR-31 is p21-dependent.

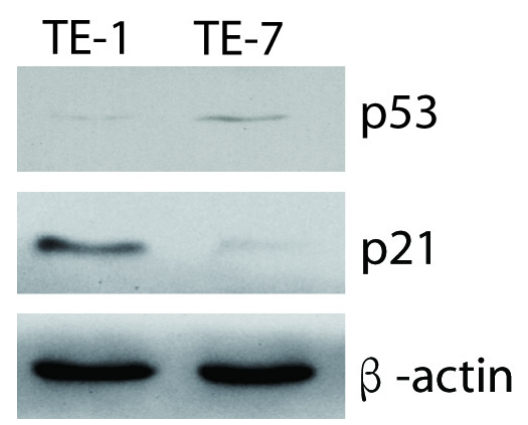

Figure 3: p21 was highly expressed in TE-1 cells and cannot be detected in TE-7 cells. Western blot of endogenous p21 and p53 expression in TE- 1 and TE-7 cells was shown. $\beta$-actin was used as internal control.

\section{DISCUSSION}

In this study, we found that miR-31 inhibits viability, colony formation and invasion in ESCC cells. This inhibitory effect appeared to be a result of downregulation of at least two downstream miR-31 target oncogenes, E2F2 and STK40. Of interest, miR-31 exerted its antitumor effects only in a cell line expressing low p21 levels, whereas the inhibitory effects of miR-31 in cells with high levels of p21 were largely silenced and could be rescued by 21 shRNA treatment. This finding was confirmed in 27 tumor tissues derived from ESCC patients by real time RT-PCR. Pearson correlation analysis for the expression profiles of miR-31, two target oncogenes, and p21 suggested that tumor cells with low p21 levels were candidates for future therapeutic delivery of miR-31.

miR-31 is a pleiotropic molecule that can act as either an oncogene or a tumor suppressor in different cancer types, although it is usually classified as a tumor suppressor $[11,25]$. As in other cancer types, the function of miR-31 in esophageal cancer is controversial. While some reports identify its oncogenic potential in esophageal cancers $[17,18]$, Zhao et al. [20] found that high levels of miR-31 are associated with a better prognosis. Furthermore, Lynam-Lennon et al. [19] found that miR-31 was significantly reduced in esophageal tumors and downregulated in radio-resistant esophageal adenocarcinoma cells. Ectopic re-expression of miR-31 re-sensitizes radio-resistant cells to radiation, suggesting that miR-31 exhibits a tumor-suppressing function in esophageal cancer [19]. One explanation for the pleiotropism of miR-31 in different cancers was presented in an elegant study by Creighton et al. [11], wherein the authors demonstrated that the tumor-suppressive function of miR-31 in ovarian cancer was dependent on loss of p53, i.e., miR-31 only inhibited ovarian tumor cells expressing non-functional p53 [11]. In the present study, we found that in addition to regulation by $\mathrm{p} 53$, the inhibitory effect of miR-31 in ESCC was dependent on p21 deficiency. Our results suggest that the requirement for p53-deficiency in order for miR-31 to function as a tumor suppressor is likely due to a dependence on p21 deficiency.

p21 is a cyclin-dependent kinase $(\mathrm{Cdk})$ regulator that controls cell division and cell fate [26]. This gene actively participates in the regulation of genes involved in growth arrest, senescence, and aging [27, 28]. p21 is known to function as a double-edged sword in cancers [29]. It is usually regarded as a repressor in cancers, since it is a downstream target of p53 and inhibits cell cycle progression, thereby inducing senescence. However, some studies actually imply that p21 is an oncogene [30, 31]. Several reports indicate that increased p21 expression is associated with tumor progression and a poor prognosis in prostate [32, 33], ovarian [34], cervical [35], breast [36] and esophageal squamous cell carcinomas [37], as well as in brain tumors [38]. In the present study, we showed 

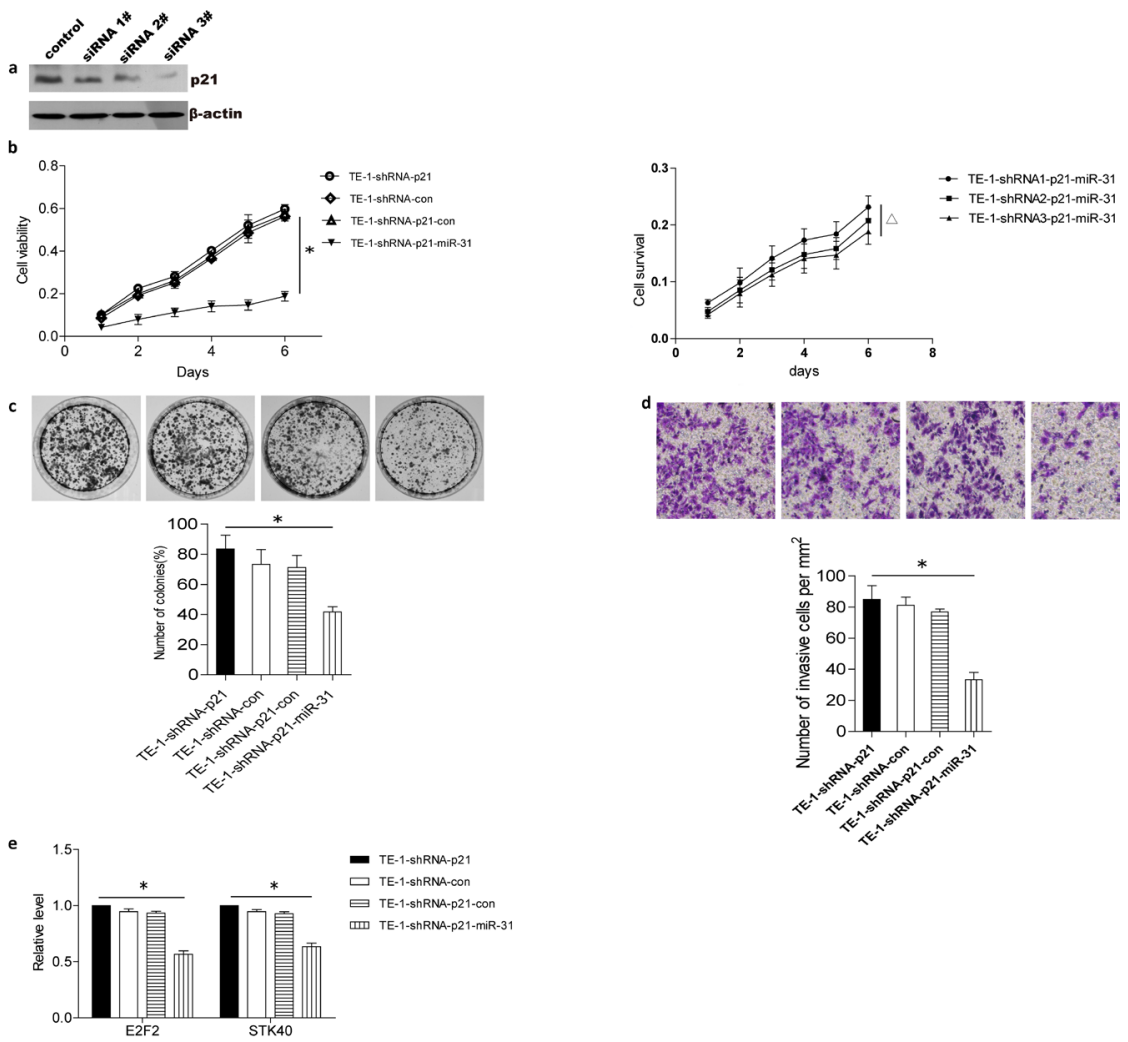

Figure 4: Inhibitory effects of miR-31 can be rescued in TE-1 cells by p21 shRNA treatment. (A) p21 expression was silenced by shRNA in TE-1 cells, as demonstrated by western blot. $3^{\#}$ shRNA was selected to do the following experiment. (B) MTT assay showed silencing of p21 in TE-1 cells permitted miR-31 overexpression to inhibit proliferation (left). Moreover, suppressive effect of miR-31 depended on silenced effect of p21 in TE-1 (right). (C) Colony formation assay indicated overexpression of miR-31 inhibited colony formation of TE-1 after p21 silencing. (D) Cell invasion assays showed overexpression of miR-31 inhibited invasion of TE-1 after p21 silencing. (E) miR-31 downregulated miR-31 target genes, E2F2 and STK-40, after p21 silencing. * indicating P $<0.05$. Statistical comparisons were made between TE-1-shRNA-p21-con vs. TE-1-shRNA-p21-miR-31, $\Delta$ indicating P $<0.05$. Statistical comparisons were made between TE-1-shRNA1-p21-miR-31 and TE-1-shRNA2-p21-miR-31 or TE-1-shRNA2-p21-miR-31 and TE-1-shRNA3-p21miR-31. Data were average of three independent experiments and was presented as means $\pm \mathrm{SEM}$ with * indicating $\mathrm{P}<0.05$. con, control. *, TE-1-con-shRNA-con v.s. TE-1-miR-31-shRNA-p21.

a
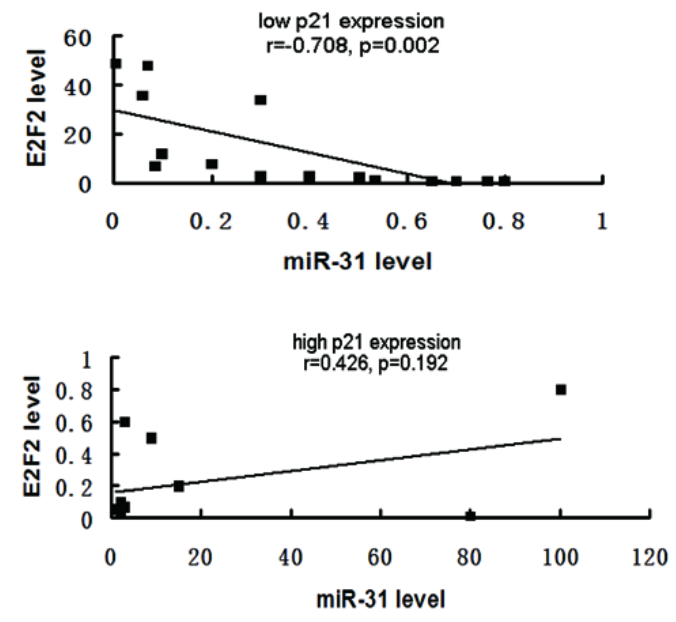

b

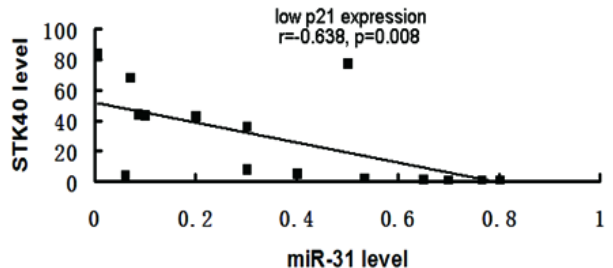

d

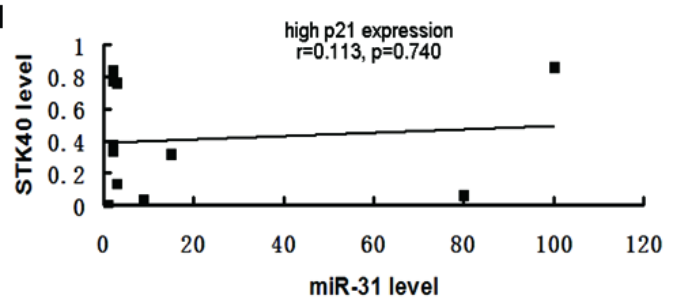

Figure 5: Correlation between miR-31 and its targets, E2F2 and STK40, in patients with ESCC was p21-dependent. mRNA of biopsies from patients with ESCC were extracted and subjected to real time RT-PCR to assay the expression of miR-31, p21, E2F2 and STK40. Patients were grouped by the expression of p21. (A). When p21 expression was low, miR-31 was negatively correlated with its target genes, E2F2 and STK40, as shown by Pearson correlation analysis. (B). When p21 expression was high, miR-31 was not correlated with its target genes. 
that $\mathrm{p} 21$ regulates the tumor-suppressive function of miR31 in ESCC. When p21 is highly expressed, miR-31 does not inhibit expression of miR-31 downstream target genes, nor does miR-31 inhibit proliferation, colony formation, or invasion. One explanation of this phenomenon is the possibility that p53 mutation led to low p21 expression and changed susceptibility to cancer treatment [39, 40]. Our Pearson correlation analysis of the expression levels of miR-31, its target oncogenes, and p21 in 27 ESCC tissues suggested that miR-31 can regulate its target genes only in p21-low patients.

Although p21 is usually regarded as a downstream effector gene of p53 and is believed to be rarely mutated [41], high-throughput gene chip assays have recently suggested that p21 expression varies among cancer cell lines and cancer tissues, including esophageal squamous cell cancers [42-44]. Moreover, p21 is regulated not only by $\mathrm{p} 53$, but also by other regulators such as MTA1 [45], E2F1 [46] or several nuclear receptors [47] including retinoid receptors, vitamin $\mathrm{D}$ receptors and androgen receptors. Thus, future studies are required for exploring the molecular mechanisms by which p21 regulates the tumor suppressive function for miR-31 and potential interventions to disrupt the $\mathrm{p} 21$-mediated suppression of miR-31 for treatment of ESCC patients.

In summary, we demonstrate that miR-31 exhibits inhibitory effects in ESCC, in a p21-dependent manner (summarized in Fig. 6), providing a novel mechanism for the tumor suppressor function of miR-31. From a translational perspective, our data suggest that in the future, miR-31 delivery therapy may benefit ESCC patients with low p21 expression, constituting a potential target for personalized medicine.

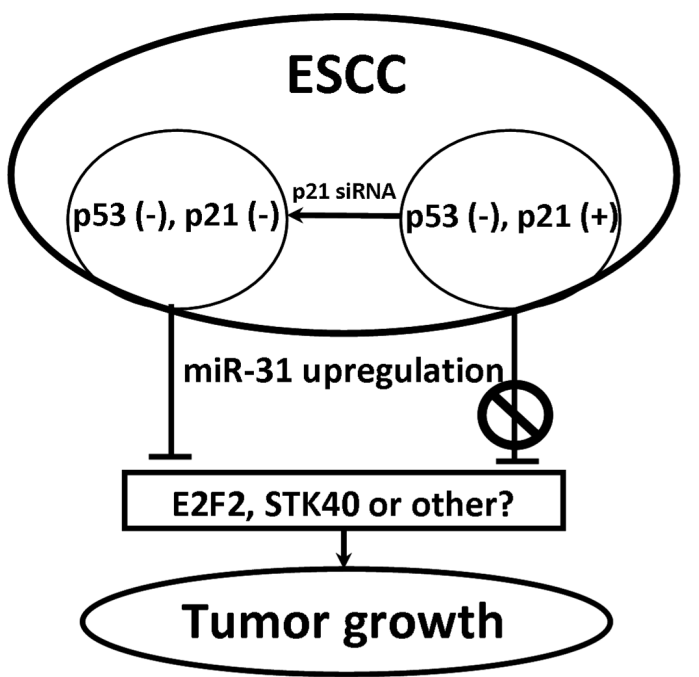

Figure 6: Schematic diagram of p21 dependence of tumor suppression by miR-31 in ESCC.

\section{MATERIALS AND METHODS}

\section{Clinical specimens}

Twenty-seven pairs of primary ESCC tumors were obtained from 27 patients (median age at diagnosis, 60.6 years; range from 38 to 79 years). Matched normal adjacent tissues were defined as tissues located at least 1.0 $\mathrm{cm}$ apart from the visible tumor lesions. Specimens were deposited in RNALater (Qiagen, Germany) or snap-frozen in liquid nitrogen and subsequently stored at $-80^{\circ} \mathrm{C}$. All patients underwent esophagectomy without preoperative chemotherapy or radiotherapy in the Affiliated Cancer Hospital of Shantou University Medical College between November of 2010 and May of 2011. This study was approved by the Ethics Committee of Shantou University Medical College, and written informed consent was obtained from all patients.

\section{Cell lines}

Human esophageal squamous cell carcinoma TE-1 and TE-7 cell lines were kindly provided by Dr. X. C. Xu (UT M.D. Anderson Cancer Center, USA) and cultured in high glucose DMEM supplemented with 10\% fetal bovine serum (Gibco), $100 \mathrm{U} / \mathrm{ml}$ penicillin and $100 \mu \mathrm{g} /$ $\mathrm{ml}$ streptomycin at $37^{\circ} \mathrm{C}$ in a humidified atmosphere containing $5 \% \mathrm{CO}_{2}$, and passaged when cells reached nearly $80 \%$ confluence.

\section{Overexpression of miR-31}

TE-7 or TE-1 cells were transfected in six-well plates $\left(2.5 \times 10^{5}\right.$ per well) using $7.5 \mu \mathrm{L}$ Lipofectamine 2000 transfection reagent (Invitrogen) and $3 \mu \mathrm{g}$ hsamiR-31 mimic (90 nmol/L; Dharmacon) according to manufacturer's instructions. Control groups of cells were treated with transfection reagent alone (mock transfection). Cells were harvested $48 \mathrm{~h}$ after transfection, and E2F2 and STK40 were tested by real time RT-PCR. For functional assays including proliferation, colony formation and invasion measurements, CMV-TurboRFPmiR-31-IRES-puro (Open Biosystems) was packaged and used to infect the TE-7 or TE-1 cell lines, according to the manufacturer's instructions. The DNA plasmid carrying a non-targeting sequence (Open Biosystems) was used as a negative control. Forty-eight hours post-transfection, virus-containing media was filtered $(0.45 \mu \mathrm{m})$ and added onto TE-7 or TE-1 cells with appropriate dilution in the presence of polybrene $(8 \mu \mathrm{g} / \mathrm{mL})$. The virus-containing media was changed with fresh cell medium $6 \mathrm{~h}$ postinfection. The infection efficiency was measured by examining the cells under fluorescence microscope and 
was determined to be $>95 \%$.

\section{shRNA knockdown of p21 in TE-1 cells}

Stable transfection was performed at about $80 \%$ confluence in 24-well plates using Lipofectamine LTX and Plus Reagents (Invitrogen) according to the manufacturer's instructions. Briefly, a total of $2 \times 10^{5}$ TE-1 cells were inoculated into each well in high glucose DMEM containing 10\% FBS without antibiotics. p2 1 shRNA (shp21) or control shRNA $(0.1 \mathrm{mg})$ (mock) (Santa Cruz Biotechnology) vectors were transfected with $0.5 \mathrm{ml}$ of Plus Reagents and $1.25 \mathrm{ml}$ of Lipofectamine LTX. Interference sequences for p21 shRNA were listed at supplementary table 1 . After transfection, cells were isolated in culture medium containing $2 \mathrm{mg} / \mathrm{ml}$ puromycin (Invitrogen). After 3 to 4 weeks, resistant cell colonies were isolated and transferred to 6-well plates, and gradually expanded to $10-\mathrm{cm}$ dishes. At $90 \%$ confluence, western blot analyses were performed to assess the efficiency of p21 knockdown.

\section{MTT assay}

Cell proliferation was measured with a colorimetric assay reagent, thiazolyl blue tetrazolium Bromide (MTT, Sigma-Aldrich, China). Absorbance was read at $590 \mathrm{~nm}$ with a reference filter of $620 \mathrm{~nm}$.

\section{Transwell invasion assay}

Cell invasion assays were performed using transwells. Briefly, $1 \times 10^{4}$ cells were inoculated in a 24well transwell unit on polycarbonate filter with $8 \mu \mathrm{m}$ pores (Costar, Cambridge, MA) coated with Matrigel (Becton Dickinson, Franklin Lakes, NJ). After 24-h incubation at $37 \mathrm{oC}$, cells that had passed through the filter were stained with Giemsa and scored for the number.

\section{Colony formation assay}

For colony formation, cells $\left(2 \times 10^{3}\right)$ were trypsinized and cultured in $60-\mathrm{mm}$ culture dishes. The dishes were incubated for two weeks, then colonies were stained with $0.1 \%$ crystal violet, then photographed and counted.

\section{Western blot assay}

As previously described [48], cells were lysed in RIPA buffer. The proteins were separated by SDS-PAGE and then transferred to PVDF membranes (Millipore,
Bedford, MA). Blots were probed with antibodies against p53 (1:500, Santa Cruz, Dallas, TX), p21 (1:1000, Cell Signaling, Danvers, MA), $\beta$-actin (1:5000, Sigma, St. Louis, MO). After washing, blots were incubated with horseradish peroxidase-conjugated secondary antibodies and visualized using an enhanced chemiluminescence kit (Pierce, Rockford, IL).

\section{Real time RT-PCR assay}

Cells or tissues were harvested with Trizol Reagent (Invitrogen, Carlsbad, CA, USA) and total RNA was isolated according to the manufacturer's instructions. cDNA synthesis was performed using the Superscript III RT-PCR kit (Invitrogen). Real-time PCR was performed using a Cepheid SmartCycler II (Sunnyvale, CA, USA) with gene-specific real-time PCR primers. Specifically, stem-loop real-time RT-PCR was used to analyze the expression of miR-31. Relative quantification (RQ) of selected genes and miR-31 expression was normalized with respect to GAPDH and U6 respectively. Corresponding adjacent esophageal tissues were used as calibrator samples. The expression of the target gene was calculated using the equation $2-\Delta \mathrm{Ct}$, where $\Delta \mathrm{Ct}=(\mathrm{Ct}$ target gene $-\mathrm{Ct}$ reference gene). The relative expression of target genes in carcinoma tissue was calculated by 2- $\Delta \Delta \mathrm{Ct}$, where $\Delta \Delta \mathrm{Ct}=(\Delta \mathrm{Ct}$ target gene in the tumor tissue $-\Delta \mathrm{Ct}$ target gene in the adjacent normal tissue). Data were presented as $\log 10$ of the relative quantification equal to the fold-change of gene expression in ESCC tissue compared to its corresponding adjacent esophageal tissue. High or low p21 expression was defined as its expression was higher or lower compared to corresponding adjacent esophageal tissue. The primers for PCR are list in supplementary table 2 .

\section{Statistical analysis}

Comparisons of real time data were analyzed by the unpaired $t$ test, whereas qualitative data were analyzed by the chi-square test. Correlation was determined by Pearson correlation analysis. All statistical analyses were performed and visualized by GraphPad Prism 5.0. A P $<0.05$ was considered statistically significant. All experiments were performed in triplicate and repeated twice.

\section{ACKNOWLEDGEMENTS}

This work was partly supported by National Natural Science Foundation of China, 81071736, 30973508 (H. Zhang). 


\section{Author contributions}

H.Zhang. contributed to study concept and design, obtained funding, administrative support and study supervision. Z.N., F.L.G. F.L. and Q.L. contributed to performance of experiments and acquisition of data. Z.N., T.T., B.Z., G.F.L. and H.Zhu. analysis and interpretation of data, drafting the article and statistical analysis. G.W.L., S.C. and D.H. contributed to techinical or material support. H.Zhu. M.S. and H.Zhang. contributed to critical revision of the article. All authors reviewed the manuscript.

\section{Disclosure of Potential Conflicts of Interest}

No potential conflicts of interest were disclosed.

\section{Abbreviations}

ESCC, esophageal squamous cell cancer; miR-31, microRNA-31; p21, cyclin-dependent kinase inhibitor 1; E2F2, E2F transcription factor 2; STK40, serine/threonine kinase 40; RT-PCR, reverse transcriptase-polymerase chain reaction.

\section{REFERENCES}

1. S. B. Umar, D. E. Fleischer, Esophageal cancer: epidemiology, pathogenesis and prevention. Nature Clinical Practice Gastroenterology \& Hepatology 5, 517 (2008).

2. A. Jemal, F. Bray, M. M. Center, J. Ferlay, E. Ward, D. Forman, Global cancer statistics. CA: a cancer journal for clinicians 61, 69 (2011).

3. Y. Feng, C. Ke, Q. Tang, H. Dong, X. Zheng, W. Lin et al., Metformin promotes autophagy and apoptosis in esophageal squamous cell carcinoma by downregulating Stat3 signaling. Cell Death Dis 5, e1088 (2014).

4. P. C. Enzinger, R. J. Mayer, Esophageal cancer. New England Journal of Medicine 349, 2241 (2003).

5. T. Lerut, W. Coosemans, G. Decker, P. De Leyn, P. Nafteux, D. Van Raemdonck, Cancer of the esophagus and gastroesophageal junction: potentially curative therapies. Surgical oncology 10, 113 (2001).

6. H. Zhang, W. Lin, K. Kannan, L. Luo, J. Li, P. W. Chao et al., Aberrant chimeric RNA GOLM1-MAK10 encoding a secreted fusion protein as a molecular signature for human esophageal squamous cell carcinoma. Oncotarget 4, 2135 (2013)

7. Z. Guohong, S. Min, W. DuenMei, H. Songnian, L. Min, L. Jinsong et al., Genetic heterogeneity of oesophageal cancer in high-incidence areas of southern and northern China. PloS one 5, e9668 (2010).

8. Y.-J. You, Y.-P. Chen, X.-X. Zheng, S. J. Meltzer, H. Zhang, Aberrant methylation of the PTPRO gene in peripheral blood as a potential biomarker in esophageal squamous cell carcinoma patients. Cancer Lett 315, 138 (2012).

9. Q. Tang, G. Li, X. Wei, J. Zhang, J. F. Chiu, D. Hasenmayer et al., Resveratrol-induced apoptosis is enhanced by inhibition of autophagy in esophageal squamous cell carcinoma. Cancer Lett 336, 325 (2013).

10. Y. Hu, A. M. Correa, A. Hoque, B. Guan, F. Ye, J. Huang et al., Prognostic significance of differentially expressed miRNAs in esophageal cancer. International journal of cancer 128, 132 (2011).

11. C. J. Creighton, M. D. Fountain, Z. Yu, A. K. Nagaraja, H. Zhu, M. Khan et al., Molecular profiling uncovers a p53-associated role for microRNA-31 in inhibiting the proliferation of serous ovarian carcinomas and other cancers. Cancer research 70, 1906 (2010).

12. S. Valastyan, A. Chang, N. Benaich, F. Reinhardt, R. A. Weinberg, Activation of miR-31 function in alreadyestablished metastases elicits metastatic regression. Genes \& development 25, 646 (2011).

13. O. Aprelikova, X. Yu, J. Palla, B.-R. Wei, S. John, M. Yi et al., The role of miR-31 and its target gene SATB2 in cancerassociated fibroblasts. Cell Cycle 9, 4387 (2010).

14. P.-C. Lin, Y.-L. Chiu, S. Banerjee, K. Park, J. M. Mosquera, E. Giannopoulou et al., Epigenetic repression of miR-31 disrupts androgen receptor homeostasis and contributes to prostate cancer progression. Cancer research 73, 1232 (2013).

15. D. Sun, F. Yu, Y. Ma, R. Zhao, X. Chen, J. Zhu et al., MicroRNA-31 activates the Ras pathway and functions as an oncogenic microRNA in human colorectal cancer by repressing RAS p21 GTPase activating protein 1 (RASA1). Journal of Biological Chemistry 288, 9508 (2013).

16. Y.-H. Tsai, M.-F. Wu, Y.-H. Wu, S.-J. Chang, S.-F. Lin, T. V. Sharp et al., The M type K15 protein of Kaposi's sarcomaassociated herpesvirus regulates microRNA expression via its SH2-binding motif to induce cell migration and invasion. Journal of virology 83, 622 (2009).

17. S. G. Liu, X. G. Qin, B. S. Zhao, B. Qi, W. J. Yao, T. Y. Wang et al., Differential expression of miRNAs in esophageal cancer tissue. Oncol Lett 5, 1639 (2013).

18. T. Zhang, Q. Wang, D. Zhao, Y. Cui, B. Cao, L. Guo et al., The oncogenetic role of microRNA-31 as a potential biomarker in oesophageal squamous cell carcinoma. Clin Sci (Lond) 121, 437 (2011).

19. N. Lynam-Lennon, J. V. Reynolds, L. Marignol, O. M. Sheils, G. P. Pidgeon, S. G. Maher, MicroRNA-31 modulates tumour sensitivity to radiation in oesophageal adenocarcinoma. J Mol Med (Berl) 90, 1449 (2012).

20. B. S. Zhao, S. G. Liu, T. Y. Wang, Y. H. Ji, B. Qi, Y. P. Tao et al., Screening of microRNA in patients with esophageal cancer at same tumor node metastasis stage with different prognoses. Asian Pac J Cancer Prev 14, 139 (2013).

21. Y. Yang, R. S. Tarapore, M. H. Jarmel, M.-P. Tetreault, J. P. Katz, p53 mutation alters the effect of the esophageal tumor 
suppressor KLF5 on keratinocyte proliferation. Cell Cycle 11, 4033 (2012).

22. S. Komatsu, I. Imoto, H. Tsuda, K. I. Kozaki, T. Muramatsu, Y. Shimada et al., Overexpression of SMYD2 relates to tumor cell proliferation and malignant outcome of esophageal squamous cell carcinoma. Carcinogenesis 30, 1139 (2009).

23. C. Barnas, G. Martel-Planche, Y. Furukawa, M. Hollstein, R. Montesano, P. Hainaut, Inactivation of the p53 protein in cell lines derived from human esophageal cancers. International journal of cancer 71, 79 (1997).

24. N. Xu, F. Meisgen, L. M. Butler, G. Han, X.-J. Wang, C. Söderberg-Nauclér et al., MicroRNA-31 Is overexpressed in psoriasis and modulates inflammatory cytokine and chemokine production in keratinocytes via targeting serine/ threonine kinase 40. The Journal of Immunology 190, 678 (2013).

25. S. Valastyan, F. Reinhardt, N. Benaich, D. Calogrias, A. M. Szász, Z. C. Wang et al., A pleiotropically acting microRNA, miR-31, inhibits breast cancer metastasis. Cell 137, 1032 (2009).

26. B. P. Zhou, Y. Liao, W. Xia, B. Spohn, M.-H. Lee, M.-C. Hung, Cytoplasmic localization of p21Cip1/WAF1 by Aktinduced phosphorylation in HER-2/neu-overexpressing cells. Nat Cell Biol 3, 245 (2001).

27. B.-D. Chang, K. Watanabe, E. V. Broude, J. Fang, J. C. Poole, T. V. Kalinichenko et al., Effects of p21Wafl/ Cip1/Sdi1 on cellular gene expression: implications for carcinogenesis, senescence, and age-related diseases. Proceedings of the National Academy of Sciences 97, 4291 (2000)

28. Y. Cang, J. Zhang, S. A. Nicholas, A. L. Kim, P. Zhou, S. P. Goff, DDB1 is essential for genomic stability in developing epidermis. Proceedings of the National Academy of Sciences 104, 2733 (2007).

29. T. Abbas, A. Dutta, p21 in cancer: intricate networks and multiple activities.Nat Rev Cancer 9, 400 (2009).

30. I. B. Roninson, Oncogenic functions of tumour suppressor p $21<\sup >$ Waf1/Cip $1 /$ Sdi $1</$ sup $>$ : association with cell senescence and tumour-promoting activities of stromal fibroblasts. Cancer Lett 179, 1 (2002).

31. A. L. Gartel, Is p21 an oncogene? Mol Cancer Ther 5, 1385 (2006).

32. G. Baretton, U. Klenk, J. Diebold, N. Schmeller, U. Löhrs, Proliferation-and apoptosis-associated factors in advanced prostatic carcinomas before and after androgen deprivation therapy: prognostic significance of p21/WAF1/CIP1 expression. Br J Cancer 80, 546 (1999).

33. S. Aaltomaa, P. Lipponen, M. Eskelinen, M. Ala-Opas, V. Kosma, Prognostic value and expression of p21 (waf1/cip1) protein in prostate cancer. Prostate 39, 8 (1999).

34. G. Ferrandina, A. Stoler, A. Fagotti, F. Fanfani, R. Sacco, A. De Pasqua et al., p21WAF1/CIP1 protein expression in primary ovarian cancer. Int J Oncol 17, 1231 (2000).
35. T. H. Cheung, K. W. Lo, M. M. Yu, S. F. Yim, C. S. Poon, T. K. Chung et al., Aberrant expression of p21(WAF1/CIP1) and p27(KIP1) in cervical carcinoma. Cancer Lett 172, 93 (2001).

36. C. Ceccarelli, D. Santini, P. Chieco, C. Lanciotti, M. Taffurelli, G. Paladini et al., Quantitative p21WAF - 1/p53 immunohistochemical analysis defines groups of primary invasive breast carcinomas with different prognostic indicators. International journal of cancer 95, 128 (2001).

37. M. Sarbia, H. E. Gabbert, Modern pathology: prognostic parameters in squamous cell carcinoma of the esophagus. Recent Results Cancer Res 155, 15 (2000).

38. J. M. Jung, J. M. Bruner, S. Ruan, L. A. Langford, A. P. Kyritsis, T. Kobayashi et al., Increased levels of p21WAF1/ Cip1 in human brain tumors. Oncogene 11, 2021 (1995).

39. Q. Qi, H. Zhang, W. Xu, Z. Chen, L. Wang, X. Dong et al., p21 Waf1/Cip1 polymorphisms and risk of esophageal cancer. Ann Surg Oncol 17, 1453 (2010).

40. M. Noushin Taghavi, M. Masoud Sotoudeh, N. Taghavi, F. Biramijamal, M. R.Abbaszadegan, H. Khademi et al., P21 (waf1/cip1) gene polymorphisms and possible interaction with cigarette smoking in esophageal squamous cell carcinoma in northeastern Iran: a preliminary study. Arch Iran Med 13, 235 (2010).

41. M. Shiohara, W. S. el-Deiry, M. Wada, T. Nakamaki, S. Takeuchi, R. Yang et al., Absence of WAF1 mutations in a variety of human malignancies. Blood 84, 3781 (1994).

42. M. J. Garnett, E. J. Edelman, S. J. Heidorn, C. D. Greenman, A. Dastur, K. W. Lau et al., Systematic identification of genomic markers of drug sensitivity in cancer cells. Nature 483, 570 (2012).

43. N. Hu, C. Wang, D. Ng, R. Clifford, H. H. Yang, Z. Z. Tang et al., Genomic characterization of esophageal squamous cell carcinoma from a high-risk population in China. Cancer Res 69, 5908 (2009).

44. J. Barretina, G. Caponigro, N. Stransky, K. Venkatesan, A. A. Margolin, S. Kim et al., The Cancer Cell Line Encyclopedia enables predictive modelling of anticancer drug sensitivity. Nature 483, 603 (2012).

45. D.-Q. Li, S. B. Pakala, S. D. N. Reddy, K. Ohshiro, S.-H. Peng, Y. Lian et al., Revelation of p53-independent Function of MTA1 in DNA Damage Response via Modulation of the p21WAF1-Proliferating Cell Nuclear Antigen Pathway. Journal of Biological Chemistry 285, 10044 (2010).

46. A. L. Gartel, F. Najmabadi, E. Goufman, A. L. Tyner, A role for E2F1 in Ras activation of p21 (WAF1/CIP1) transcription. Oncogene 19, 961 (2000).

47. A. L. Gartel, A. L. Tyner, Transcriptional regulation of the p21((WAF1/CIP1)) gene. Exp Cell Res 246, 280 (1999).

48. H. Dong, H. Guo, L. Xie, G. Wang, X. Zhong, T. Khoury et al., The metastasis-associated gene MTA3, a component of the Mi-2/NuRD transcriptional repression complex, predicts prognosis of gastroesophageal junction adenocarcinoma. PloS one 8, e62986 (2013). 